\title{
Psychiatric profiles and patterns of cerebral blood flow in focal epilepsy: interactions between depression, obsessionality, and perfusion related to the laterality of the epilepsy
}

E Bettina Schmitz, John Moriarty, Durval C Costa, Howard A Ring, Peter J Ell, Michael R Trimble

\begin{abstract}
Objectives-In a study of patients with focal epilepsy the hypothesis was explored that different measurements of psychopathology are related to specific distributions of cerebral perfusion.

Methods-Forty patients had SPECT performed with ${ }^{99 \mathrm{~m}}$ Tc-HMPAO. In addition, patients received a psychiatric evaluation with the following psychiatric questionnaires: the Beck depression inventory, the Leyton obsessionality inventory, the Bear-Fedio questionnaire, and the social stress and support interview. Patients were analysed in two groups according to the laterality of the epilepsy. Nine patients were excluded based on poor quality scans $(n=1)$, unlateralised epilepsy $(n=4)$, and left or ambidextrous handedness $(n=4)$.

Results-There were no overall differences between the left and right epilepsy groups on measures of psychopathology. Associations were found between scores on some of the rating scales and regional cerebral blood flow. Specifically, for patients with left sided epilepsy, higher scores on the Beck depression inventory were associated with lower contralateral temporal and bilateral frontal perfusion, and higher occipital perfusion. For patients with right sided epilepsy higher scores on the Leyton obsessionality inventory were associated with increased perfusion in ipsilateral temporal, thalamic, and basal ganglia regions and bilateral frontal regions.

Conclusion-The results do not support the notion that lateralised epileptogenic lesions are associated with different levels of depression, obsessionality, or personality traits. They support the view that certain psychopathological symptom patterns are related to specific regional dysfunctions depending on the laterality of a hemispheric lesion.
\end{abstract}

(F Neurol Neurosurg Psychiatry 1997;62:458-463)

Keywords: focal epilepsy; laterality; HMPAO-SPECT; depression; obsessive-compulsive disorder

Behavioural changes in patients with epilepsy have been identified for many years. These range from depression and anxiety ${ }^{1}$ to psy- choses, ${ }^{2}$ and may include some specific personality problems that have variously been referred to as the interictal personality disorder of epilepsy and the Gastaut-Geschwind syndrome. ${ }^{3}$ A specific association between these syndromes and temporal lobe epilepsy has been claimed. ${ }^{45}$ This has not been easy to establish, however, and psychopathology is likely also to reflect such factors as seizure severity, global cerebral damage, medication effects, and adequacy of psychosocial supports.

The personality syndrome of epilepsy has been particularly controversial and, although Bear and $\mathrm{Fedio}^{6}$ developed their questionnaire specifically to monitor behavioural problems associated with temporal lobe disorders, it has been criticised as being a general measure of psychopathology, ${ }^{7}$ with little specificity.

It has become possible to explore brainbehaviour relations in neuropsychiatric disorders using new brain imaging techniques. Disordered cerebral function has been described in schizophrenia, depression, and obsessive-compulsive disorder using both PET and single photon emission computed tomography (SPECT).$^{4}$ In these states, mesial temporal regions as well as the frontal lobes and basal ganglia are often highlighted. A good example is the work of Baxter et al, ${ }^{8}$ who have identified changes in frontal-basal ganglia circuits in patients with obsessive-compulsive disorder. These changes have been confirmed by others ${ }^{4}$ and, furthermore, are altered with treatment. ${ }^{910}$

Interictal SPECT in patients with focal epilepsies reliably show hypometabolism and reduced cerebral blood flow associated with the site of the epileptogenic focus. ${ }^{1112}$ However, we and others have emphasised the widespread nature of perfusion changes seen in association with temporal lobe epilepsy, predominantly in limbic frontal regions. ${ }^{12}{ }^{13}$ These may be related to functional deafferentation, interictal inhibitory activity, postictal depletion of substrates, or other phenomena. ${ }^{13}$ Epilepsy is a cerebral disorder with more widespread disturbance of cerebral function than often appreciated, which may be reflected in a wide variety of behaviours, from a seizure, to an automatism, to interictal cognitive and behavioural symptoms. It is possible that the distribution of localised hypometabolism is associated with the interictal cognitive and behaviour states.

In this study we explore some of these rela- 
tions further, using epilepsy as a model and SPECT as our imaging method. Our main hypotheses are: (1) affective symptoms are associated with decreased perfusion in frontotemporal regions; (2) obsessive compulsive behaviour is associated with increased perfusion in frontotemporal and basal ganglia regions; (3) personality symptoms identified by the Bear-Fedio questionnaire are associated with decreased perfusion in the temporal lobe; and (4) social variables are not associated with localised cerebral perfusion.

In addition to temporal, frontal, and basal ganglia regions we also measured blood flow in the parietal and occipital cortex. These were used as control regions and we predicted finding no associations between perfusion and psychopathology in these regions. We investigated patients with left and right sided epilepsies separately and predicted that perfusion patterns would relate to psychopathology regardless of the laterality of the epilepsy.

\section{Methods}

PATIENTS

We investigated 40 consecutive patients who were referred to the National Hospital for Neurology and Neurosurgery, London for assessment and treatment of chronic focal epilepsies. One patient was subsequently excluded as a result of a parainjection of the isotope (resulting in a very poor quality scan). The clinical diagnosis of epilepsy was made by experienced epileptologists according to the International Classification of Epilepsies and Epileptic Syndromes. ${ }^{14}$ None of the patients were being investigated specifically for psychiatric illness, although some had had previous episodes of psychopathology. Patients are described in more detail elsewhere. ${ }^{12}$ The project was approved by the ethics committee of the National Hospital for Neurology and Neurosurgery.

\section{LATERALITY OF EPILEPSY}

The laterality of the epilepsy was established on the basis of EEG and MRI findings. Seventeen patients had ictal EEGs in addition to interictal EEGs. All patients had MRI (Siemens, 1.5 Tesla). The laterality of EEG and MRI findings were independently determined by investigators who were blind to the SPECT and psychiatric data. Handedness was assessed with the handedness research questionnaire. ${ }^{15}$

\section{SPECT STUDIES}

We used ${ }^{99 m}$ Tc-HMPAO SPECT (mean dose $606 \mathrm{MBq}$ ). Patients were scanned interictally with their eyes closed using a triple detector SPECT system (GE Neurocam, high resolution collimators, 128 projections, $64 \times 64$ matrix, total acquisition time 28 minutes). Slices of $9 \mathrm{~mm}$ thickness were reconstructed in coronal, sagittal, and transverse planes using a filtered back projection method. The transverse planes were specially oriented along the axis of the temporal lobe. The resolution of the scanner at FWHM is $9 \cdot 2 \mathrm{~mm} .{ }^{16}$
Scans were quantitatively analysed by an observer who was blind to clinical data and psychiatric findings. For quantification we used a region of interest method; $4 \mathrm{~cm}^{2}$ box regions of interest ( $5 \times 5$ pixel, $3.6 \mathrm{~cm}^{3}$ voxel) were placed in the following regions: mesial temporal, lateral temporal, orbitofrontal, dorsolateral frontal, thalamus and parietal cortex on coronal slices, basal ganglia and occipital cortex on horizontal slices, and frontopolar cortex on sagittal slices. Regions were identified visually from the SPECT with reference to an atlas of brain anatomy. ${ }^{17}$ To allow intersubject comparisons, for all regions of interest we estimated an activity index (AI):

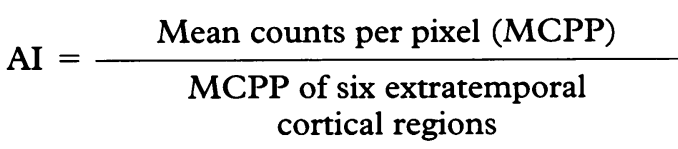

The six extratemporal cortical regions were right and left dorsolateral frontal cortex, right and left parietal cortex, and right and left occipital cortex

\section{PSYCHIATRIC QUESTIONNAIRES}

All patients were given the following questionnaires on the day of the SPECT: the Beck depression inventory, the Leyton obsessional inventory, the Bear-Fedio questionnaire, and the social stress and support interview.

The Beck depression inventory is a 21 item self rating scale with four severity ratings for each item. Each item describes a specific behaviour manifestation of depression. ${ }^{18}$ The Leyton obsessional inventory is a comprehensive rating scale that evaluates both state and trait obsessive-compulsive symptoms and consists of 68 questions. We only considered the total (state plus trait) score for the purpose of this analysis. ${ }^{19}$ Average scores of 18 (SD 10) have been reported in normal populations with a tendency to score slightly lower on repeat testing. Obsessional patients score much higher (44 (SD 11))..$^{20}$ The Bear-Fedio questionnaire is a self administered 18 item questionnaire relating to issues thought to reflect the interictal personality syndrome of epilepsy, such as religiosity, hypergraphia, circumstantiality, viscosity, paranoia, and altered sexuality. ${ }^{6}$ There are no standardised scores for this scale. The social stress and support interview ${ }^{21}$ is a semistructured interview to rate social stress and support, consisting of six sections which are each given ratings between -2 and +2 . We included this as a "control" questionnaire because there are no hypotheses with respect to localised abnormalities relating to social problems. All patients underwent formal psychological testing and both verbal and performance IQs were estimated.

\section{MEASURES OF ASSOCIATION}

Because laterality of the epilepsy focus itself is likely to affect measures of perfusion and may also influence psychopathology, patients were divided into those whose epilepsy could be lateralised to the left or the right. For clarity, patients in whom the epilepsy could not clearly be lateralised were excluded as well as patients 
Table 1 Scores on psychiatric rating scales in patients with right and left sided epilepsy

\begin{tabular}{|c|c|c|c|c|}
\hline & $\begin{array}{l}\text { All patients } \\
\text { Mean (SD, range) }\end{array}$ & $\begin{array}{l}\text { Right epilepsy } \\
\text { Mean (SD, range) }\end{array}$ & $\begin{array}{l}\text { Left epilepsy } \\
\text { Mean (SD, range) }\end{array}$ & Pvalue \\
\hline BDI & $\begin{array}{l}10 \cdot 8 \\
(13 \cdot 1,0-71)\end{array}$ & $\begin{array}{l}12 \cdot 6 \\
(16 \cdot 7,0-71)\end{array}$ & $\begin{array}{l}8 \cdot 3 \\
(4 \cdot 9,2-17)\end{array}$ & 0.38 \\
\hline LOI & $\begin{array}{l}22 \cdot 7 \\
(11 \cdot 31,2-54)\end{array}$ & $\begin{array}{l}25 \cdot 6 \\
(13 \cdot 4,2-54)\end{array}$ & $\begin{array}{l}18 \cdot 5 \\
(5 \cdot 8,7-26)\end{array}$ & 0.09 \\
\hline $\mathrm{BFQ}$ & $\begin{array}{l}4 \cdot 0 \\
(3 \cdot 1,0-12)\end{array}$ & $\begin{array}{l}4 \cdot 7 \\
(3 \cdot 7,0-12)\end{array}$ & $\begin{array}{l}3 \cdot 2 \\
(2 \cdot 1,0-8)\end{array}$ & $0 \cdot 21$ \\
\hline SSSI & $\begin{array}{l}1 \cdot 1 \\
(2 \cdot 79,-6-+5)\end{array}$ & $\begin{array}{l}1 \cdot 5 \\
(2 \cdot 5,-4-+5)\end{array}$ & $\begin{array}{l}0.5 \\
(0 \cdot 3,-6-+4)\end{array}$ & 0.33 \\
\hline VIQ & $\begin{array}{l}92 \cdot 4 \\
(13 \cdot 8,70-123)\end{array}$ & $\begin{array}{l}93 \cdot 1 \\
(14 \cdot 2,70-123)\end{array}$ & $\begin{array}{l}91 \cdot 5 \\
(13.9,70-107)\end{array}$ & 0.78 \\
\hline PIQ & $\begin{array}{l}93 \cdot 3 \\
(16 \cdot 5,64-122)\end{array}$ & $\begin{array}{l}92 \cdot 1 \\
(17 \cdot 0,64-122)\end{array}$ & $\begin{array}{l}94 \cdot 8 \\
(16 \cdot 5,67-113)\end{array}$ & 0.68 \\
\hline
\end{tabular}

*Unpaired $t$ test, two tailed, test for equality.

$\mathrm{BDI}=$ Beck depression inventory; $\mathrm{LOI}=$ Leyton obsessionality inventory; $\mathrm{BFO}=$ Bear-Fedio questionnaire; SSSI $=$ social stress and support interview; VIQ $=$ Verbal IQ; PIQ $=$ performance IQ.

Table 2 Patients with left sided epilepsy $(n=13)$ : correlations between regional blood flow (activity index) and scores on psychiatric rating scales

\begin{tabular}{|c|c|c|c|c|c|}
\hline$R O I$ & & $B D I$ & LOI & $B F Q$ & SSSI \\
\hline Mesial temporal & Right & -0.19 & -0.19 & 0.41 & $-0 \cdot 11$ \\
\hline Lateral temporal & Right & $-0 \cdot 62^{\star}$ & -0.15 & $0 \cdot 16$ & 0.50 \\
\hline Orbitofrontal & Right & 0.37 & 0.23 & 0.43 & $0 \cdot 11$ \\
\hline Frontopolar & Right & $-0 \cdot 80^{\star \star}$ & -0.01 & $0 \cdot 26$ & 0.51 \\
\hline Dorsolateral frontal & Right & $-0 \cdot 73^{\star \star}$ & -0.35 & 0.33 & 0.06 \\
\hline Basal ganglia & Right & -0.04 & 0.03 & 0.04 & -0.04 \\
\hline Thalamus & Right & -0.12 & 0.06 & $0 \cdot 14$ & 0.03 \\
\hline Parietal & Right & -0.36 & 0.06 & 0.53 & -0.20 \\
\hline Occipital & Right & $0 \cdot 60^{\star}$ & $0 \cdot 08$ & $-0 \cdot 20$ & 0.32 \\
\hline Mesial temporal & Left & -0.08 & $0 \cdot 29$ & 0.30 & 0.17 \\
\hline Lateral temporal & Left & $-0 \cdot 12$ & 0.35 & 0.29 & 0.42 \\
\hline Orbitofrontal & Left & -0.8 & 0.06 & $0 \cdot 28$ & 0.32 \\
\hline Frontopolar & Left & $-0.89^{\star \star \star}$ & 0.07 & $0 \cdot 21$ & 0.36 \\
\hline Dorsolateral frontal & Left & $-0 \cdot 71^{\star}$ & $-0 \cdot 30$ & $-0 \cdot 25$ & 0.09 \\
\hline Basal ganglia & Left & $-0 \cdot 19$ & $0 \cdot 16$ & 0.04 & -0.21 \\
\hline Thalamus & Left & -0.02 & 0.13 & -0.01 & 0.12 \\
\hline Parietal & Left & $0 \cdot 30$ & 0.39 & -0.08 & -0.46 \\
\hline Occipital & Left & $0 \cdot 62^{\star}$ & 0.01 & -0.40 & $0 \cdot 17$ \\
\hline
\end{tabular}

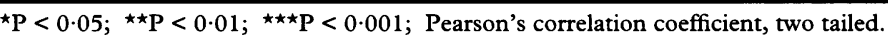

who were not right handed. The Pearson's product moment correlation coefficient was then calculated for each selected cerebral region and each measure of behaviour. All statistical analyses were two tailed. Because of the exploratory nature of these studies, we did not apply strict Bonferroni corrections. However, to compensate for multiple testing, the significance level was set at 0.01 , although a statististical trend at a level of 0.05 is indicated in the tables. The SPSS/PC+ computer programme (v4) was used for these calculations.

\section{Results}

The EEG was non-localising in seven cases. Of the remaining patients, 31 had a fron-

Table 3 Patients with right sided epilepsy $(n=18)$ : correlations between regional blood flow (activity index) and scores on psychiatric rating scales

\begin{tabular}{|c|c|c|c|c|c|}
\hline$R O I$ & & $B D I$ & $L O I$ & $B F Q$ & SSSI \\
\hline Mesial temporal & Right & $0 \cdot 25$ & $0.56^{\star}$ & $0 \cdot 22$ & -0.40 \\
\hline Lateral temporal & Right & $0 \cdot 11$ & $0 \cdot 65^{\star \star}$ & $0 \cdot 20$ & $-0 \cdot 13$ \\
\hline Orbitofrontal & Right & 0.06 & $0 \cdot 62^{\star \star}$ & 0.19 & -0.30 \\
\hline Frontopolar & Right & $0 \cdot 24$ & 0.43 & 0.28 & -0.33 \\
\hline Dorsolateral frontal & Right & $0 \cdot 14$ & 0.17 & $0 \cdot 20$ & $-0 \cdot 13$ \\
\hline Basal ganglia & Right & -0.08 & $0 \cdot 54^{\star}$ & 0.06 & -0.07 \\
\hline Thalamus & Right & $0 \cdot 21$ & $0 \cdot 56^{\star}$ & $0 \cdot 14$ & $-0 \cdot 15$ \\
\hline Parietal & Right & 0.31 & $0 \cdot 11$ & -0.32 & -0.25 \\
\hline Occipital & Right & $0 \cdot 24$ & 0.00 & 0.06 & $0 \cdot 24$ \\
\hline Mesial temporal & Left & 0.26 & $0 \cdot 30$ & $0 \cdot 26$ & -0.22 \\
\hline Lateral temporal & Left & $0 \cdot 10$ & $0 \cdot 27$ & $0 \cdot 21$ & $-0 \cdot 15$ \\
\hline Orbitofrontal & Left & -0.03 & $0.58^{\star}$ & 0.35 & -0.07 \\
\hline Frontopolar & Left & $0 \cdot 23$ & $0 \cdot 50^{\star}$ & 0.35 & -0.28 \\
\hline Dorsolateral frontal & Left & 0 & $0 \cdot 14$ & $0 \cdot 19$ & -0.35 \\
\hline Basal ganglia & Left & 0.03 & 0.46 & $-0 \cdot 12$ & $0 \cdot 19$ \\
\hline Thalamus & Left & $0 \cdot 15$ & 0.45 & 0.08 & 0.04 \\
\hline Parietal & Left & $0 \cdot 18$ & -0.23 & $-0 \cdot 15$ & $-0 \cdot 17$ \\
\hline Occipital & Left & 0.33 & $-0 \cdot 16$ & -0.02 & 0.44 \\
\hline
\end{tabular}

${ }^{\star} \mathrm{P}<0.05 ;{ }^{\star \star} \mathrm{P}<0.01 ;$ Pearson's correlation coefficient, two tailed totemporal focus and one had an occipital focus. Brain MRI was normal in seven patients, 28 had hippocampal pathology, three had temporal pathology without hippocampal involvement, and one had an extratemporal structural abnormality.

In 35 patients the epilepsy could be lateralised to one side based on abnormalities on EEG, MRI, or both. In two patients, who were subsequently excluded, EEG and MRI indicated opposite sides. Another two patients who had negative or non-lateralising findings in both EEG and MRI were excluded. We also excluded two patients who were left handed and two patients who were ambidextrous. Eighteen patients with right sided epilepsy and 13 patients with left sided epilepsy remained for further analyses.

The mean age of onset of epilepsy was $10 \cdot 6$ (SD 6.9, range 1-26) years, and the mean age at investigation was $29 \cdot 1$ (SD $9 \cdot 2$, range 16-56) years. Thirteen patients were male, 18 female. All patients were taking antiepileptic drugs. None was receiving medication for psychiatric indications.

Table 1 gives the scores on the rating scales. There was no relation between the scores and sex, age of onset of epilepsy, or age at investigation. Scores did not significantly differentiate patients with right or left sided epilepsy.

There was a significant positive correlation between scores on the Leyton obsessionality inventory and the Bear-Fedio questionnaire $(r=0.53, \mathrm{P}<0.01)$. Comparing the right : left ratios of blood flow in patients with right versus patients with left sided epilepsy there was significant ipsilateral hypoperfusion of the mesial temporal lobe ( $t$ test for unequality, $P<0.05)$.

MEASURES OF ASSOCIATION (TABLES 2 AND 3) Patients with left sided epilepsy showed a negative association between scores on the Beck depression inventory and contralateral lateral temporal lobe perfusion and bilateral frontal perfusion (highly significant for the left frontopolar cortex), and a positive association between scores on the Beck depression inventory and occipital cortex perfusion.

Patients with right sided epilepsy showed a positive association between scores on the Leyton obsessionality inventory and ipsilateral temporal lobe, thalamic and basal ganglia perfusion, and bilateral frontal perfusion.

There were no significant correlations between scores on both the Bear-Fedio questionnaire and the social stress and support interview, and regional cerebral blood flow.

\section{Discussion}

METHODOLOGICAL CONSIDERATIONS

The scanner used by our group is an up to date, triple headed camera, with high resolution. In epilepsy we have shown that this has good specificity with regard to laterality of the seizure focus. ${ }^{12}$ With respect to quantification of perfusion it must be acknowledged that acitivity indices can only approximate absolute blood flow. ${ }^{12}$ Our method of region of interest 
placement has been shown to have validity and reliability in past investigations. ${ }^{22}$

In presenting the data, we have relied on correlation analysis, and draw attention to profiles of perfusion found in association with various rating scale scores. Although multiple analyses have been performed, strict Bonferroni corrections have not been applied, as we are primarily interested at this stage in exploring possible patterns of interactions and correlations, rather than individual localisation. Thus it is conceivable that some of our apparently significant correlations could have arisen by chance, although not all of them. Of course, there is no way of knowing which ones might and which ones might not be spurious.

Epilepsy has been used by us as a biological model of cerebral dysfunction with which to explore brain-behaviour relations. Our patients had various seizure types, and syndromes, and were not selected because they had. However, the psychiatric illnesses are common in epilepsy, and a high frequency of reported symptoms may be expected in this population. As these patients did not have a systematic psychiatric evaluation, we cannot exclude the possibility that some of them may have satisfied diagnostic criteria for a psychiatric syndrome. Although further evaluation of their mental states from a clinical viewpoint would have been useful, our intention was to study interrelations between levels of perfusion and psychiatric symptoms, as opposed to specific diagnostic categories.

The range of scores on the rating scales allow us to explore the associations between particular behavioural syndromes in epilepsy and patterns of regional blood flow. For example, although only two of our patients scored greater than 30 on the Beck depression inventory (indicating a severe degree of depression), almost half of them $(n=17)$ scored greater than 10 , suggesting at least milder degrees of mood disturbance not amounting to psychiatric disorder. In three comparable studies on focal epilepsy, the mean Beck depression inventory scores were lower than in our study, being 6,8 , and 9 respectively. ${ }^{132324}$ There are no comparable data with respect to scores on the Leyton obsessionality inventory and scores on the social stress and support interview. As noted above, there are few data available on the Bear-Fedio questionnaire, and in the original paper mean data are not provided.

\section{LATERALITY OF EPILEPSY, MOOD, AND} REGIONAL PERFUSION

For the depression scores, a relation with regional perfusion abnormalities was found with the left sided epilepsies only, the higher the ratings for depression, the lower the perfusion in frontal areas bilaterally. This finding of an association between mood and frontal dysfunction is in keeping with the data from others who have used neurological models to explore the genesis of affective symptoms. For example, the results of Starkstein snd Robinson ${ }^{25}$ in stroke patients, and of Ring et $a l^{26}$ in patients with Parkinson's disease emphasise an association between affect and frontal areas. It also supports data from patients with affective disorder and no underlying neurological lesions, in whom several groups have noted frontal hypoperfusion. ${ }^{27} 28$

In epilepsy, Hermann et al ${ }^{24}$ noted a specific relation between levels of perseveration, suggestive of frontal lobe involvement, and depression, but only in patients with a left sided focus. These findings were confirmed and extended in a recent study by Seidenberg $e t$ al. ${ }^{23}$

An association between left hemispheric lesions and depressive symptoms has been noted previously. Specifically, in relation to epilepsy, many-for example, Mendez et $a l^{29}$ - but not all investigators have found this relation. In a study of depression and epilepsy, Robertson et $a l^{1}$ were unable to find any specific links between depression and the localisation of the epilepsy focus. We found similar levels of self reported depression for patients with left and right unilateral epilepsy.

This is in accordance with the findings of Seidenberg et al ${ }^{23}$ who suggested that specific cortical regions in both hemispheres asymmetrically are involved in depression. Yamaguchi et $a l^{30}$ in a study on blood flow and depression in poststroke patients, found that severity of depression was inversely correlated with blood flow in the parieto-occipital regions of the right hemisphere and in anterior temporal regions of the left hemisphere. Heller ${ }^{31}$ and Robinson et al $^{32}$ have discussed further the evidence supporting the possibility of two distinct lateralised neural systems influencing mood state, a left frontal and a right posterior system. The studies of Hermann et $a l^{24}$ and Seidenberg et $a l^{23}$ were unable to investigate this hypothesis because they only used the Wisconsin card sorting test as a measurement for frontal dysfunction. We included posterior regions in our analysis, and are able to test the hypothesis of an additional posterior mood regulating system which may be involved in patients with right hemispheric lesions. Our data do not support these suggestions, as we found no hypoperfusion changes in these regions.

Ross and Rush ${ }^{33}$ suggested that endogenous depressions are predominantly modulated by the right hemisphere, whereas the more cognitive non-endogenous depressions are modulated by the left hemisphere. The Beck depression inventory is heighly weighted toward cognitive aspects of depression rather than the syndrome of major depression. Frontal lobe hypoperfusion in this context may reflect the effects of cortical rather than subcortical dysfunction, leading to cognitive rather than vegetative manifestations. ${ }^{13}$ Thus one explanation of the lack of any localised perfusion pattern in our right sided epilepsy group might be on account of our use of the Besk depression inventory, as opposed to another method of assessing affective symptoms. In addition, prominent endogenous and vegetative features, which may be related more to diencephalic abnormalities, cannot be studied by present HMPAO-SPECT or PET technologies. 
The relative occipital hyperperfusion, seen only in patients with left sided pathology, is consistent with several findings that a posterior shift in perfusion may occur in depression. ${ }^{34}$

LATERALITY OF EPILEPSY, OBSESSIVE

COMPULSIVE BEHAVIOUR, AND REGIONAL PERFUSION

Despite the reporting of single cases ${ }^{35}{ }^{36}$ a specific link between epilepsy and obsessive-compulsive disorder has not been shown. However, it is known that several neurological states are clinically associated with a range of obsessive-compulsive disorder-like phenomena, perhaps better described as obsessivecompulsive behaviour. These include encephalits lethargica, Sydenham's chorea, and Parkinson's disease. ${ }^{374}$ In descriptions of the personality of patients with epilepsy, some obsessional traits have been identified, and some of these are included in the Bear-Fedio questionnaire. We found a significant association between subject scores on the the Leyton obsessionality inventory and the Bear-Fedio questionnaire.

For our perfusion data, the most obvious associations with the Leyton obsessionality inventory were right temporal and bilateral frontal, with additional associations being seen with the measurements of right thalamic and bilateral basal ganglia perfusion. These findings are of particular interest in view of the PET findings in obsessive-compulsive disorder, ${ }^{838}{ }^{39}$ which include frontal, caudate, and thalamic hyperperfusion. It is important to note that the changes in cerebral perfusion associated with the Leyton obsessionality inventory do not directly reflect the pathology of the seizure focus, as interictal scans in patients with focal epilepsies show reduced cerebral blood flow associated with the site of the epileptogenic focus. ${ }^{12}$

Our finding that the links between perfusion and obsessive-compulsive disorder were restricted to the right epilepsy group were unexpected but nevertheless intriguing. It does not seem that the measure of obsessionality is linked to the presence of an epilepsy focus on the right side, as we found no difference in our scores for patients with right or left sided lesions. Rather, our data support the hypothesis that specific cerebral perfusion patterns are seen in association with our measure of obsessionality. We found this association only in those patients whose epilepsy had a right sided focus. Our data do show a clear distinction between the perfusion changes linked with the Leyton obsessionality inventory and those with the Beck depression inventory. The first reflect mainly hyperperfusion, the second hypoperfusion.

PERSONALITY AND SOCIAL VARIABLES AND PATTERN OF PERFUSION

Our study failed to show significant associations between regional blood flow and the total score on the Bear-Fedio questionnaire. This finding is consistent with some previous studies which rejected Bear and Fedio's original findings that there is a specific constellation of personality traits related to temporal lobe pathology. ${ }^{70}$

We did not find specific perfusion patterns associated with the social stress and support interview. As this was deliberately chosen as a control questionnaire, containing items which seemed unlikely to be related to the localisation of the epileptogenic lesion, the absence of any significant correlations strengthens our positive findings for the measures of psychopathology.

\section{Conclusions}

Studies of psychopathology in the absence of a neurological illness have increasingly considered symptoms or syndromes as opposed to diagnostic categories - for example, the associations between psychomotor retardation and abnormalities of dorsolateral prefrontal cortex perfusion described by Bench et al. ${ }^{27}$ Our data support this approach. The finding in our study of different patterns of blood flow in patients with epilepsy arising from different sites, and the fact that these patterns can be associated with selective psychopathological symptoms, is of special interest to those interested in behavioural neurology. In particular we emphasise that perfusion changes seen distant from a focal site of pathology, which reflect on distributed networks of abnormality, may be closely linked to the changes in behaviour reported in patients with neurological lesions, including epilepsy.

The relations we found between depressed mood and frontal hypoperfusion and between obsessionality and frontal hyperperfusion add to the accumulating data supporting a prominent role for frontal brain regions in the regulation of mood and behaviour. ${ }^{4}$ Our data also support the reported associations between obsessive-compulsive disorder and cerebral circuits involving frontal-subcortical distributed pathways.

1 Robertson MM. The organic contribution to depressive illness in patients with epilepsy. Fournal of Epilepsy 1989; 2:189-230.

2 Trimble MR. The psychoses of epilepsy. New York: Raven Press, 1991.

3 Trimble MR. Interictal psychoses of epilepsy. In: Smith DB, Treiman DM, Trimble MR, eds. Advances in neurology. New York: Raven Press, 1991:143-52.

4 Trimble MR. Biological psychiatry. 2nd ed. Chichester: John Wiley, 1996.

5 Waxman SG, Geschwind N. The interictal behaviour syndrome in temporal lobe epilepsy. Arch Gen Psychiatry drome in temporal

6 Bear DM, Fedio P. Quantitative analysis of interictal behaviour in temporal lobe epilepsy. Arch Neurol 1977; 34:454-67.

7 Mungas D. Interictal behaviour abnormality in temporal lobe epilepsy. A specific syndrome or non-specific psychopathology? Arch Gen Psychiatry 1982;39:108-11.

8 Baxter LR, Schwartz JM, Maziotta JC, et al. Cerebral glucose metabolic rates in obsessive compulsive disorder. Am f Psychiatry 1988;145:1560-3.

9 Hoehn-Saric R, Pearlson GD, Harris GJ, Machlin SR, Camargo EE. Effects of fluoxetine on regional blood flow in obsessive compulsive patients Am $\mathcal{f}$ Psychiatry 1991;148:1243-5.

10 Baxter LR, Schwartz JM, Bergman JM, et al. Caudate glucose metabolic rate changes with both drug and behaviour therapy for obsessive compulsive disorder. Arch Gen Psychiatry 1992;49:681-9.

11 Duncan R, Patterson J, Hadley DM, et al. CT, MR, and SPECT imaging in temporal lobe epilepsy. $\mathcal{f}$ Neurol Neurosurg Psychiatry 1990;53:11-5.

12 Schmitz B, Costa DC, Jackson DG, Moriarty J, Duncan JS, Trimble MR, Ell PJ. Optimised interictal HMPAO-
Thitz B, Costa DC, Jackson DG, Moriarty J, Duncan JS, Trimble MR, Ell PJ. Optimised interictal HMPAO-
SPECT in the evaluation of partial epilepsy. Epilepsy Res 1995;21:159-67. 
13 Bromfield EB, Altshuler L, Leiderman DB, et al. Cerebral metabolism amd depression in patients with complex partial seizures. Arch Neurol 1992;49:617-23.

14 Commission on Classification and Terminology of the International League Against Epilepsy. Proposal for revised classification of epilepsies and epileptic synrevised classification of epilepsies
dromes. Epilepsia 1989;30:389-99.

15 Annett M. The binomial distribution of right, mixed and left handedness. $Q \mathcal{F}$ Exp Psychol $A$ 1967;19:327-33.

16 Kouris K, Jarritt PH, Costa DC, Ell PJ. Physical assessment of the GE/CGR Neurocam and comparison with a single rotating gamma-camera. Eur f Nucl Med 1992;9: $236-42$.

17 Tailarach J, Tournoux P. Co-planar stereotaxic atlas of the human brain. Stuttgart: Thieme Verlag, 1988.

18 Beck AT, Ward CH, Mendelson M, Mock J, Erbaugh J. An inventory for measuring depression. Arch Gen Psychiatry 1961;4:561-71.

19 Snowdon J. A comparison of the written and postbox forms of the Leyton obsessional inventory. Psychol Med 1980; 10:165-70.

20 Cooper J. The Leyton obsessional inventory. Psychol Med 1970;1:48-64.

21 Jenkins R, Mann AH, Belsey E. The background, design and use of a short interview to assess social stress and and use of a short interview to assess social stress and support in resear

22 Ring HA. Functional imaging in neuropsychiatric disorders [MD thesis]. London: University of London, 1993.

23 Seidenberg M, Hermann B, Noe A, Wyler AR. Depression in temporal lobe epilepsy: interaction between laterality of lesion and Wisconsin card sort performance. Neuropsychiatry, Neuropsychology, and Behavioural Neurology 1995;8:81-7.

24 Hermann BP, Seidenberg M, Haltiner A, Wyler AR. Mood state in unilateral temporal lobe epilepsy. Biol Psychiatry 1991;30:1205-18.

25 Starkstein SE, Robinson RG. Affective disorders and cerebral vascular disease. Br f Psychiatry 1989;154:170-82.

26 Ring HA, Bench C, Trimble MR. Depression in Parkinson's disease. Br f Psychiatry 1994;165:333-9.

27 Baxter LR, Schwartz JM, Phelps ME, et al. Reduction of prefrontal cortex glucose metabolism common to three types of depression. Arch Gen Psychiatry 1989;46:243-50. 28 Bench CJ, Friston KJ, Brown RG, Frackowiak RS, Dolan RJ. Regional cerebral blood flow in depression: relationship to clinical dimensions. Psychol Med 1993;23:579-90.

29 Mendez MF, Taylor JL, Doss RC, Salguero P. Depression in secondary epilepsy: relation to lesion laterality. $\mathcal{f}$ Neurol Neurosurg Psychiatry 1994;57:232-3.

30 Yamagushi S, Kobayashi S, Koide H, Tsunematsu T Longitudinal study of regional cerebral blood flow changes in depression after stroke. Stroke 1992;23: changes in

31 Heller W. The neuropsychology of emotion: developmental patterns and implications for psychopathology. In: Stein WL, Leventhal B, Trabasso T, eds. Psychological and biological approaches to emotion. Hillsdale, NJ: Lawrence Erlbaum Associates, 1990.

32 Robinson RG, Starr LB, Price TR. A two year longitudina study of mood disorders following stroke. Br F Psychiatry 1984;144:256-62.

33 Ross ED, Rush J. Diagnosis and neuroanatomical correlates of depression in brain-damaged patients. Arch Gen Psychiatry 1981;38:1344-54.

34 Bench CJ, Friston KJ, Brown RG, Scott LC, Frackowiak RS, Dolan RJ. The anatomy of melancholia-focal abnormalities of cerebral blood flow in major depression. abnormalities of cerebral blood

$35 \mathrm{Kettl}, \mathrm{PA}$, Marks IM. Neurological factors in obsessivecompulsive disorder. Two case reports and a review of the literature. Br $\mathcal{F}$ Psychiatry $1986 ; 149: 315-9$.

36 Kroll L, Drummond LM. Temporal lobe epilepsy and obsessive compulsive symptoms. F Nerv Ment Dis 1983; 181:457-8.

37 Cummings JL. Frontal-subcortical circuits and human behaviour. Arch Neurol 1993;50:873-80.

38 McGuire PK, Bench CJ, Frith CD, et al. Functiona anatomy of obsessive-compulsive phenomena. $\mathrm{Br} \mathscr{f}$ Psychiatry 1994;164:459-68.

39 Rauch SL, Jenike MA, Alpert NM, et al. Regional CBF measured during symptom preoccupation in OCD using 51:62-70.

40 Rodin E, Schmaltz S. The Bear-Fedio personality inventory and temporal lobe epilepsy. Neurology 1984;34:591-6. 